Über die Periode der Apsidenbewegung von Y Cygni. Von P. Guthnick.

In A. N. $4_{3} 33,202.3_{37}$, hatte ich zur Illustrierung eines vereinfachten Verfahrens, bei Doppelstern-Veränderlichen die aus Apsidenbewegung bei merklicher Exzentrizität entspringende Ungleichheit rechnerisch zu behandeln, den merkwürdigen Algolstern Y Cygni herangezogen. Es lag nicht im Rahmen meiner damaligen Mitteilung, das große Beobachtungsmaterial vor dem Gebrauch eingehender zu untersuchen, obwohl es mir nicht entgangen war, daß ganze Reihen von Epochenbestimmungen mit starken systematischen Einflüssen behaftet sein müssen. Dunér hat übrigens in seinen bekannten Arbeiten über Y Cygni bereits darauf hingewiesen. Die Periode der großen Ungleichheit, die Dunér zu 4 I. I Jahre ansetzte, schien mir nach dem seit Duners Bearbeitung neu hinzugekommenen spärlichen Beobachtungsmaterial wesentlich kürzer zu sein, war indessen immer noch nicht mit Sicherheit anzugeben. Ich nahm $34 \frac{1}{2}$ Jahre an. Nun bin ich im vergangenen Winter durch die Freundlichkeit Herrn Zinners in den Besitz von 5 neuen Minimumbestimmungen on ihm gelangt, die ich hier anfuhre:

$242145^{2.4} \mathrm{r}, 455.40,45^{8.40}, 497.35,506.30$ geoz. m.Z.Gr. Dieselben zeigen, daß die von mir angenommene Periode der Ungleichheit viel zu kurz ist und daß die wahre Periode sogar noch weit länger als díe von Dunér angenommene sein muß. Ich habe daraufhin das mir zugängliche Beobachtungsmaterial etwas eingehender geprüft und möchte das Ergebnis kurz mitteilen.

I)a nur in wenigen Fällen die Originalbeobachtungen veröffentlicht worden sind, so war es nicht möglich, den starken systematischen Einflüssen, die gerade bei diesem Stern infolge der besonderen Verhältnisse auftreten, auf den Grund zu gehen. Ich mußte mich darauf beschränken, das Material sorgfältig $z \mathrm{u}$. sichten, einzelne Reihen von Bestim. mungen, die offenbar systematisch stark verfälscht sind, von der weiteren Behandlung auszuschließen, wie es auch Duner schon getan hatte, und die übrigen Bestimmungen verschiedener Beobachter nach Möglichkeit so zusammenzufassen, daß die systematischen Einflüsse in der Hauptsache kompensiert zu werden scheinen. Wo die Originalbeobachtungen vorlagen, habe ich, wenn möglich, neue Epochen abgeleitet. Dies gilt insbesondere für die Beobachtungen Plassmanns und Wendells. Die Annahme der kurzen $34 \%$ jährigen Periode wurde wesentlich begünstigt durch das nur im absteigenden Ast beobachtete Minimum der geraden Epoche 4776 (Harv. Ann. 69. 134,1906 ), das in A. N. 4833 wohl zu früh angesetzt wurde. Die Normalepochen lauten nunmehr:

I. Gerade Minima.

$\begin{array}{crcc}\text { Epoche } & \text { Helioz.m.Z.Gr. } & \text { Zahl d. Minima } & \text { B-R } \\ 6 & 2410268.440 & 5 & -0.009 \\ 210 & 0879.753 & 4 & +0.02 \mathrm{I} \\ (476) & (1676.690) & (4) & (-0.099) \\ 531 & 1841.586 & 2 & -0.007 \\ 581 & 1991.401 & 16 & -0.014 \\ 610 & 2078.308 & 11 & -0.008 \\ 820 & 2707.577 & 19 & +0.018 \\ 1080 & 3486.612 & 5 & -0.005 \\ 1143 & 3675.392 & 3 & +0.005 \\ 1421 & 4508.369 & 5 & +0.003\end{array}$

\begin{tabular}{|c|c|c|c|}
\hline Epoche & Helioz. m.Z.Gr. & Zahl d. Minima & $B-R$ \\
\hline $17 \circ 3$ & $24 I 5353 \cdot 318$ & I I & 0.000 \\
\hline 2388 & $7405.72 \pm$ & I & $-0.01 \pm$ \\
\hline 3265 & $2420033 \cdot 3^{8} 4$ & I 3 & +0.007 \\
\hline & 2. Ungerade & Minima. & \\
\hline 95 & 2410536.536 & I I & -0.007 \\
\hline 352 & I 306.580 & I 7 & +0.014 \\
\hline 595 & 2034.649 & I 2 & $-0.00 I$ \\
\hline $\begin{array}{c}65^{6} \\
(824)\end{array}$ & $\begin{array}{c}2217.420 \\
(2720.722)\end{array}$ & $\stackrel{9}{(7)}$ & $\begin{array}{c}0.000 \\
(-0.07 \mathrm{I})\end{array}$ \\
\hline 956 & 3116.290 & 3 & -0.012 \\
\hline I 186 & $3^{805.443}$ & 8 & -0.010 \\
\hline I 435 & $455^{1.55^{2}}$ & 4 & +0.007 \\
\hline $175^{\circ}$ & 5495.429 & 5 & +0.021 \\
\hline 2267 & $7 \circ 44.58$ & $\mathbf{r}$ & +0.01 \\
\hline 3135 & 9645.510 & 5 & -0.018 \\
\hline 3745 & 2421473.375 & 5 & -0.002 \\
\hline
\end{tabular}

Die eingeklammerten Normalepochen sind für die Ableitung der Elemente nicht benutzt worden; es sind dieselben wie in A. N. 4833. Außerdem wurden die Minima 1888 Okt. 10 (Yendell), I 89 I Aug. 20 (Yendell) und I 892 März 20 (Dunér) ausgeschlossen. $\mathrm{Zu}$ den älteren Minima ist ein von Gore beobachtetes, I 89 I Okt. 30.335 , neu hinzugekommen. Nach verschiedenen Versuchen fand ich die folgenden Elemente als die zur Zeit wahrscheinlichsten:

Gerades Minimum $=2410250.406 \mathrm{~m} . \mathrm{Z}$. Gr.

$$
+2.996336 \cdot E+0^{d}{ }_{17} \text { o } \sin [0.0554(E+400)]
$$

Ungerades Minimum $=2410251.969 \mathrm{~m} . \mathrm{Z}$. Gr.

$$
+2.996336 \cdot E-0_{1}{ }^{d} 70 \sin [0.0554(E+400)] \text {. }
$$

Die Darstellung mit diesen Elementen ist unter $B-R$ gegeben. Gemäß den Lichtwechselelementen ist die Exzentrizität der Bahn 0.178, die Umlaufszeit der Apsiden 6500 Perioden oder 53.3 Jahre, der Durchgang des Periastrons durch die Gesichtslinie Epoche - 400. Die Exzentrizität ist so groß, daß die Glieder dritter Ordnung bereits merklich werden. Es wird aber gegenwärtig kaum Zweck haben, sie zu berücksichtigen. Die Beobachtungen der nächsten Jahre werden die noch vorhandene Ungewißheit bezüglich der Periode der Ungleichheit voraussichtlich beseitigen.

Im übrigen bestehen aber noch mehrere andere Fragen, die eine eingehende Untersuchung der Originalbeobachtungen wünschenswert und interessant erscheinen lassen. Während z. B. die sonstigen Umstände für die obige Festsetzung des Periastrondurchganges sprechen, sind die wenig zahlreichen, aber genauen Wendellschen Messungen von 19 10 mit dieser Festsetzung kaum in Einklang zu bringen. Die Abnahme der Helligkeit war I 906 bei der Phase - o. I 3 bereits im Gange, während sie I 9 I 0 bei der Phase -0.08 noch nicht begonnen hatte. $\mathrm{Da}$ die Reihe innerhalb des befriedigend durch die Elemente dargestellten Beobachtungsmateriales liegt, so scheint dies nicht an den Elementen $z \mathfrak{u}$ liegen. Sehr merkwürdig sind auch die Wahrnehmungen Yendells, Mai bis Juli I 893 (A. J. 3 г 2). Die Minima waren den Angaben Yendells gemäß derart flach, daß ihre Existenz kaum als gesichert gelten kann; ja, Mai 24 konnte uberhaupt keine merkliche Helligkeitsänderung festgestellt werden, obwohl die Beobachtungen 
bis in die Mitte des berechneten Minimums reichten. Es muß dahingestellt bleiben, ob hierbei systematische Einflüsse auf die Beobachtungen im Spiele waren oder eine wirkliche Störung des Lichtwechsels vorlag. Außer Knotenbewegung bei starker Neigung der Bahn gegen ihre feste Ebene wäre noch eine andere Störungsursache denkbar, die sich mir bei einem der lichtelektrisch aufgefundenen, als Bedeckungsveränderlicher angesprochenen Veränderlichen ( $\beta$ Ursae majoris) aufgedrängt hat. Wenn auf den einander zugekehrten Hemisphären der Komponenten durch die gegenseitigen physischen Einwirkungen Änderungen der Helligkeitsverteilung (z. B. infolge lokaler Schwankungen der Absorption) auftreten, die nicht mit der Periode des Lichtwechsels zu verlaufen brauchen, so kann dadurch offenbar der Verlauf der Minima von Epoche zu Epoche oder auch in längeren Zeiträumen erheblich beeinflußt werden. Unter Umständen könnten sogar BedeckungsSternwarte Berlin-Babelsberg, I 918 Juli I 6. minima vorgetäuscht werden, wo in Wirklichkeit keine merklichen Bedeckungen stattfinden, oder umgekehrt wirkliche Bedeckungen völlig maskiert werden. Ist demnach die Dauer der beobachteten Minima im Vergleich zur Periode sehr groß, so kann es zweifelhaft sein, ob überhaupt Bedeckungen vorliegen, selbst wenn die Minima mit den spektroskopischen Konjunktionen nahe zusammenfallen. Ist dagegen die Dauer der Minima im Vergleich zur Periode kurz, wie es bei Y Cygni ( $\mathrm{r}: 8 \pm$ ) und den meisten anderen Algolsternen der Fall ist, so kann wohl im allgemeinen der Charakter der Minima nicht zweifelhaft, ibr Verlauf aber nichtsdestoweniger durch physische Ursachen der angedeuteten Art verhältnismäßig erheblich beeinflußt sein. Beispiele für algolähnliche Minima von sehr wechselnder Tiefe, die wohl sicher keine Bedeckungen sind, liefert $\eta$ Geminorum. Die Dauer der Minima beträgt bei diesem Stern in der Regel etwa die Hälfte der Periode.

\section{P. Guthnick.}

\section{Photographische Helligkeitsmessungen von}

Die folgenden wenigen Beobachtungen von $T$ Vulpeculae sind ebenso wie die schon mitgeteilten zahlreicheren von $\mathrm{S}$ Sagittae (A. N. 4916, 205.28I) mit dem UV-Zeißtriplet ( $a=$ $15 \mathrm{~cm}, f=150 \mathrm{~cm}$ ) angestellt. Die Aufnahmen erfolgten $3 \mathrm{~mm}$ intrafokal bei Verwendung des auch bei S Sagittae (l.c.) benutzten Objektivgitters, für welches der Sterngrößenunterschied $z$ wischen Zentralbild und Spektren erster Ordnung om.95 beträgt. Im ganzen wurden in 35 Nächten 175 Platten in der Zeit von r 9 Io Okt. 16 bis 19 I 5 Sept. 20 erhalten. Davon sind die 8 Platten $\mathrm{Nr}$. 1787 bis 1844 inkl. von Herrn Dr. Müch aufgenommen. Die mittlere Epoche aller Beobach-

\section{T Vulpeculae 1910-15. Von E. Hertzsprung.}

tungen ist J. D. 2420106 . Verwendet wurden Schleußnerplatten, blaue Etikette. Die ersten 18 Platten vom Format $20 \times 20 \mathrm{~cm}$ enthalten je 2 Expositionen, die späteren vom Format $9 \times 12 \mathrm{~cm}$ nur eine. Die Ausmessung im Mikrophotometer wurde von Frl. $H$. Mattenklodt ausgeführt. Die Phasen sind heliozentrisch von I 9 1 0 Okt. I6, I $8^{\mathrm{h}}$ Sternzeit Potsdam $=$ J. D. 24 I 896 1. 1 46295 m. Z. Gr. als Nullpunkt aus gerechnet und in Bruchteilen der Luizetschen Periode $4^{\mathrm{d}} 4.355^{2} \mathrm{I}$ ausgedrückt. Demnach verschiebt sich die Phase für jeden Sterntag um OP.22483708. Außer T Vulpeculae wurden im Mikrophotometer die in folgender Tabelle mitaufgeführten fünf Sterne gemessen.

\begin{tabular}{|c|c|c|c|c|c|c|c|}
\hline $\begin{array}{l}\text { Bezeich- } \\
\text { nung } \\
T\end{array}$ & $\begin{array}{c}\mathrm{BD} \\
+27^{\circ} 3890\end{array}$ & $\begin{array}{c}a_{1900} \\
20^{\mathrm{h}} 47^{\mathrm{m}} \cdot 2\end{array}$ & $\begin{array}{l}31900 \\
+27^{\circ} 52^{\prime}\end{array}$ & $\begin{array}{c}\text { S } \\
\text { vis. } P D \\
\text { var. }\end{array}$ & $\begin{array}{c}\text { erngrö } \\
\text { vis. Har } \\
\text { var. }\end{array}$ & $\begin{array}{l}\text { n } \\
22-7^{m} \cdot 35\end{array}$ & $\begin{array}{l}\text { Farb.-Ind. } \\
m_{U V}-m_{P}\end{array}$ \\
\hline$a$ & +264008 & $20 \quad 45.8$ & +27 & $7^{m} \cdot 5^{2}$ & $7: 04$ & $7^{m} \cdot 30$ & $-0^{\mathrm{m}} 22$ \\
\hline$b$ & +264017 & $20 \quad 47.8$ & +2643 & 4.80 & 4.76 & 5.94 & +1.14 \\
\hline$c$ & +273909 & 2050.1 & +28 & 6.88 & 6.44 & 6.45 & -0.43 \\
\hline$d$ & +273911 & $20 \quad 50.3$ & +2741 & $5 \cdot$ I 2 & 5.24 & $7 \cdot 46$ & +2.34 \\
\hline$e$ & +273868 & $20 \quad 4.3 .6$ & +2714 & $7 \cdot 34$ & 6.95 & $7 \cdot 5^{6}$ & +0.22 \\
\hline
\end{tabular}

Die in dieser Tabelle an die UV-Sterngrößen der Sterne $a, b, c, d$ und $e$ schon angebrachten Reduktionen (Feldkorrektur) auf den Ort von $\mathrm{T}$ Vulpeculae betragen für die 5 Sterne bezw. $+0^{\mathrm{m}} 04,+0^{\mathrm{m}} .04,-0^{\mathrm{m}} \mathrm{o} 5,-0^{\mathrm{m}} .02$ und $+0^{\mathrm{m}} .03$. Um die bei den differentiellen Helligkeitsmessungen wirksamen scheinbaren Größen der Sterne auf den Platten zu erhalten, müssen diese Korrektionen wieder rückgängig gemacht werden. - Der Nullpunkt der UV-Größen ist nur angenähert richtig, d. h. für $A$-Sterne mit der visuellen Harvard-Skala übereinstimmend.

Von den beiden Sternen $d$ und $e$ wird weiter unten die Rede sein. Zum Vergleich mit $T$ Vulpeculae dienten nur die 3 Sterne $a, b$ und $c$, deren mittlere Koordinaten so nahe mit denen von $T$ Vulpeculae übereinstimmen, daß von einer Korrektion wegen Änderung der Extinktionsdifferenzen in verschiedenen Stundenwinkeln abgeseben werden konnte, indem

alle Aufnahmen zwischen I Stunde vor und 3 Stunden nach dem Meridian geschahen. In der Haupttabelle ist für jede Beobachtungsnacht die mittlere scheinbare Sterngrößendifferenz zwischen $\mathrm{T}$ Vulpeculae und den 3 Vergleichsternen $a, b$ und $c$ angegeben. Die Resultate sind außerdem in der Figur graphisch dargestellt. Die beiden Nächte J. D. 0752 und 0756 erhielten halbes Gewicht, weil sie nur je 2 Platten mit je einer Exposition lieferten. Es wurden sodann die folgenden Mittelwerte gebildet :

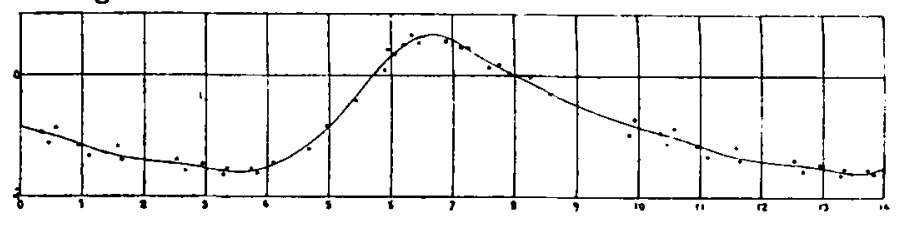

